\title{
Sex-Related Differences in Cardiac Remodeling and Reverse Remodeling After Transcatheter Aortic Valve Implantation in Patients with Severe Aortic Stenosis in a Japanese Population
}

\author{
Ryo Ninomiya, ${ }^{1}$ MD, Makoto Orii, ${ }^{2}$ MD, Jumpei Fujiwara, ${ }^{1}$ MD, Michiko Yoshizawa, ${ }^{1}$ MD, \\ Yoshifumi Nakajima, ${ }^{1}$ MD, Yu Ishikawa, ${ }^{1}$ MD, Akiko Kumagai, ${ }^{1}$ MD, \\ Tetsuya Fusazaki, ${ }^{1}$ MD, Atsushi Tashiro, ${ }^{3} \mathrm{MD}$, Hajime Kin, ${ }^{4}$ MD, \\ Kunihiro Yoshioka, ${ }^{2} \mathrm{MD}$ and Yoshihiro Morino, ${ }^{1} \mathrm{MD}$
}

\begin{abstract}
Summary
Left ventricular (LV) remodeling with aortic stenosis (AS) appears to differ according to sex, but reverse remodeling after transcatheter aortic valve implantation (TAVI) has not been elucidated in a Japanese population. This study aims to determine whether any sex-related differences in LV or reverse remodeling after TAVI exist in the context of severe AS.

Of 208 patients who received TAVI for severe AS in our institution, 100 (men, 42; mean age, $83.0 \pm 4.9$ years) underwent transthoracic echocardiography before and 3 months after TAVI. Despite similar valvular gradients, women with severe AS had lower indexed LV mass (LVMi) than did men $(152.3 \pm 35.4$ versus $173.2 \pm$ $44.6 \mathrm{~g} / \mathrm{m}^{2}, P=0.005$ ), with smaller indexed LV end-diastolic (LVEDVi) $(50.2 \pm 13.3$ versus $61.4 \pm 20.7 \mathrm{~mL} /$ $\mathrm{m}^{2}, P=0.001$ ) and end-systolic (LVESVi; $17.9 \pm 8.7$ versus $24.3 \pm 13.8 \mathrm{~mL} / \mathrm{m}^{2}, P=0.006$ ) volumes. After TAVI, women $(-6.0 \% \pm 14.4 \%)$ had higher reduction in the rate of change of relative wall thickness (RWT) than did men $(4.4 \% \pm 19.0 \%, P=0.003)$. Men $(-8.9 \% \pm 3.9 \%)$ had higher reduction in the rate of change of LVEDVi than did women $(1.5 \% \pm 3.3 \%, P=0.045)$. Incidence of $\mathrm{LV}$ reverse remodeling defined as a reduction in LVESV of $>15 \%$ was significantly higher in men $(50 \%)$ than in women $(26 \%, P=0.013)$.

In addition to sex differences in the pattern of LV remodeling with AS, reverse LV remodeling after TAVI also differed between sexes.
\end{abstract}

Key words: Heart valve diseases, LV hypertrophy, Left ventricular remodeling

(Int Heart J 2020; 61: 961-969)

A ortic stenosis (AS) is a common disease that develops regardless of race, and sex differences in this disease have been reported in various populations. ${ }^{1)}$ There is no difference in progression or prevalence by sex, but the manner of cardiac remodeling, especially left ventricular (LV) remodeling for AS, differs between the sexes..$^{2-4)} \mathrm{LV}$ remodeling is heterogeneous, with four main geometric patterns: normal geometry, concentric remodeling, concentric hypertrophy, and eccentric hypertrophy. ${ }^{5)}$ These patterns are based on LV mass (LVM), cavity size, and the ratio of these two factors. It is well known that many concentric hypertrophy changes are seen in females, while eccentric hypertrophy changes are seen in males. ${ }^{6-10)}$ Meanwhile, LV reverse remodeling after surgical aortic valve replacement (SAVR) is an important indicator related to long-term prognosis and tends to be seen in males after SAVR. ${ }^{9)}$

Transcatheter aortic valve implantation (TAVI) has been introduced in Japan and is suitable for the treatment of elderly and frail patients. The myocardial response resulting from the improvement of AS is seen clearly and quickly after TAVI. ${ }^{11)}$ However, the difference between the sexes in the change of cardiac morphology after TAVI in patients who were not indicated for SAVR has not been elucidated in a Japanese population. Therefore, this study aims to determine whether any sex-related differences exist in severe AS in terms of cardiac and reverse remodeling after TAVI in a Japanese population.

\section{Methods}

Study population and design: Between December 2013 and May 2018, 208 patients (men, 76 [36.5\%]; mean age, $83.7 \pm 4.8$ years) with severe AS undergoing TAVI at Iwate Medical University Hospital were prospectively recruited (Figure 1). During the study period, patients un-

From the ${ }^{1}$ Division of Cardiology, Department of Internal Medicine, Iwate Medical University, Iwate, Japan, ${ }^{2}$ Department of Radiology, Iwate Medical University, Iwate, Japan, ${ }^{3}$ Department of Laboratory of Medicine, Iwate Medical University, Iwate, Japan and ${ }^{4}$ Department of Cardiovascular Surgery, Iwate Medical University, Iwate, Japan.

Address for correspondence: Makoto Orii, MD, Department of Radiology, Iwate Medical University, 2-1-1, Idaidori, Yahaba, Iwate, 028-3694, Japan. E-mail: kori931@gmail.com

Received for publication March 3, 2020. Revised and accepted June 9, 2020.

Released in advance online on J-STAGE September 12, 2020.

doi: 10.1536/ihj.20-154

All rights reserved by the International Heart Journal Association. 


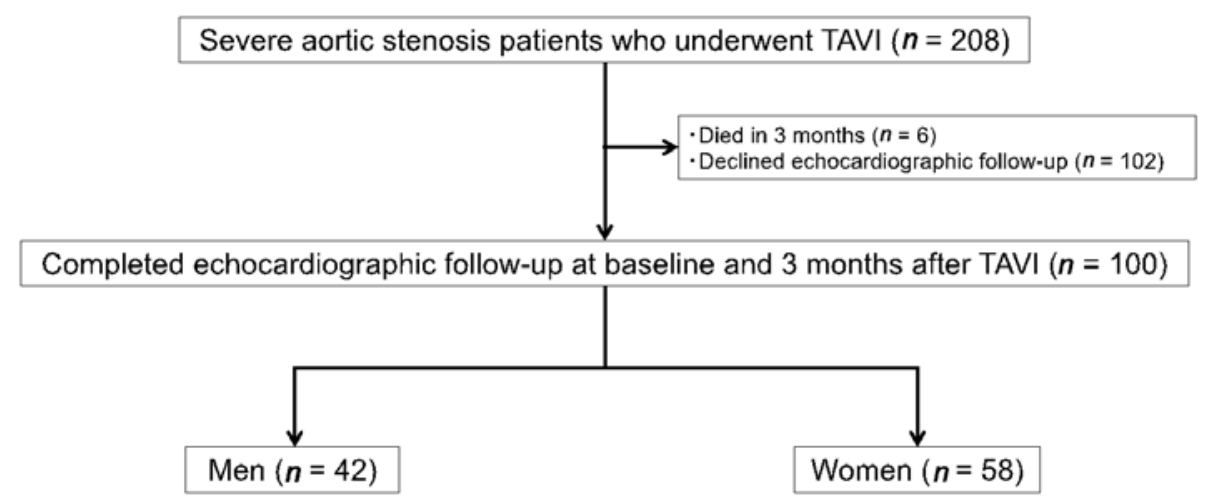

Figure 1. Patient recruitment pathway. TAVI indicates transcatheter aortic valve implantation.

derwent TAVI if they had severe AS with an aortic valve area (AVA) of $<1.0 \mathrm{~cm}^{2}$ or $<0.6 \mathrm{~cm}^{2} / \mathrm{m}^{2}$ and were deemed inoperable or at a high risk for SAVR based upon the evaluations of a dedicated multidisciplinary team. Patients were evaluated by transthoracic echocardiography before TAVI and 3 months after the procedure.

The study protocol conforms to the ethical guidelines of the 1975 Declaration of Helsinki as reflected in an $a$ priori approval by the institution's human research committee (MH2018-503), and written informed consent for data collection was obtained from each patient prior to TAVI.

TAVI procedures: Patients were selected to undergo TAVI using a transfemoral $(n=83)$, trans-aortic $(n=2)$, or trans-apical $(n=15)$ approach, depending on the size, calcification, and tortuosity of the iliofemoral arterial access. Edwards valves (Sapien-XT, Sapien 3; Edwards Lifesciences, Irvine, CA, USA) and CoreValves, Evolut-R (Medtronic, Minneapolis, MN, USA) were used, and the device size was selected according to the area derived from the mean annulus diameter calculated using multidetector computed tomography and transesophageal echocardiography. The procedures were performed by experienced interventional cardiologists according to our standard operating procedures with the patients under general anesthesia. Hemodynamic and echocardiographic parameters were continuously monitored during the procedure.

Pre- and postprocedural echocardiography: All patients underwent echocardiography before TAVI and 3 months after TAVI. All echocardiograms were performed and analyzed by the same operator on an EPIQ 7 ultrasound machine (Philips Healthcare, Inc., Andover, MA, USA) and were digitally stored and later analyzed by a researcher who was completely blinded to all clinical data. Measurements and recordings were obtained according to the American Society of Echocardiography (ASE) guidelines. ${ }^{12)}$

AVA was estimated with quantitative Doppler using the continuity equation. ${ }^{12)}$ The diameter of the LV outflow tract was measured $5 \mathrm{~mm}$ below the annulus from a zoomed image of the LV outflow tract obtained in the parasternal long-axis view. Peak flow velocity across the valve was determined in the apical window or the echocardiographic window, where the highest peak velocity could be obtained by placing the continuous wave Dop- pler cursor as close as possible to parallel with the flow across the valve. The peak transvalvular gradient was estimated using the Bernoulli equation. ${ }^{12)}$ Finally, the peak systolic flow velocity in the outflow tract was estimated with pulsed-wave Doppler.

LV wall thickness and dimensions were estimated from the average of three consecutive two-dimensional images obtained in the parasternal long-axis view according to the ASE's guidelines. ${ }^{12)}$ AS patients were categorized into four patterns of LV geometric adaption: "normal geometry," "concentric remodeling," "concentric hypertrophy," and "eccentric hypertrophy." These patterns are based on relative wall thickness (RWT), LVM, and the ratio of these two factors, as previously described. ${ }^{5)}$ RWT was calculated as the ratio of twice the interventricular septum wall thickness at end-diastole (IVSd) to the LV end-diastolic dimension (LVDd). ${ }^{13)}$ RWT was used to categorize LV hypertrophy (LVH) as either concentric (RWT $\geq 0.42$ ) or eccentric (RWT $<0.42$ ). LVM was calculated as recommended by the ASE using the Devereux formula, which incorporates LVDd, posterior wall thickness at enddiastole (PWd), IVSd, and LVDd:

$\mathrm{LVM}=0.8 \times\left(1.04\left[(\mathrm{LVDd}+\mathrm{PWd}+\mathrm{IVSd})^{3}-\right.\right.$ $\left.\left.(\mathrm{LVDd})^{3}\right]\right)+0.6$.

LVM was indexed to body surface area (BSA). LVH was defined as indexed LVM (LVMi) exceeding $110 \mathrm{~g} / \mathrm{m}^{2}$ in women and $125 \mathrm{~g} / \mathrm{m}^{2}$ in men. ${ }^{12)} \mathrm{LV}$ concentric remodeling was defined as a normal LVMi with RWT $\geq 0.42$, eccentric LVH as increased LVMi with RWT $<0.42$, and concentric LVH as increased LVMI with RWT $\geq 0.42 .{ }^{5)}$

The LV volume was measured using the biplane area length method and corrected for BSA. The LV ejection fraction (LVEF) was calculated using the biplane modified Simpson's method. Indexed left atrial volume (LAVi) was measured in LV end systole in the frame preceding mitral valve opening. Mitral inflow was assessed in the apical four-chamber view using pulsed-wave Doppler with the sample volume paced at the tips of the mitral leaflets during diastole. From the mitral inflow profile, the E- and Awave peak velocities and deceleration time were measured. Paravalvular and transvalvular regurgitation were assessed according to the Valve Academic Research Consortium-2 criteria. ${ }^{14}$ Severe patient-prosthesis mismatch (PPM) was defined as an indexed effective orifice area $0.65 \mathrm{~cm}^{2} / \mathrm{m}^{2} 3$ months after TAVI. We evaluated the 
changes in echocardiographic parameters before and after TAVI using the rate of change. The rate of change was calculated as (post-TAVI - pre-TAVI) / pre-TAVI $\times 100$ $(\%)$. We defined LV reverse remodeling as a reduction in LVESV of $>15 \% .^{15)}$

Statistical analysis: All statistical analyses were performed using $\mathrm{JMP}^{\circledR} 13$ (SAS Institute Inc., Cary, NC, USA). Normality was checked using the Shapiro-Wilk test. Continuous variables are expressed as mean \pm SD or median and interquartile range as appropriate, whereas qualitative variables are expressed as numbers and percentages. Differences between means were evaluated using paired (for before and after comparisons) and unpaired (for independent group comparisons) Student t-tests for normally distributed data and Mann-Whitney or Wilcoxon signed-rank tests for nonparametric data. The chi-square test was used for categorical variables and Fisher exact test for categorical variables with low frequencies (expected cell count $<5$ ). Pearson correlation coefficients were used to investigate the relationship of cardiac reverse remodeling parameters with baseline parameters. A twotailed $P<0.05$ was considered statistically significant.

\section{Results}

Population: Of 208 patients referred for possible enrollment into this study, 108 were excluded: 6 patients died within 3 months of causes unrelated to the TAVI procedure and echocardiography was not able to be performed at the 3-month follow-up in 102 patients. The remaining 100 patients (male, 42; female, 58) fulfilled the study criteria and were enrolled accordingly (Figure 1). There were no significant differences between the group that completed the 3-month echocardiography and that did not in age $(83.0 \pm 4.9$ versus $84.3 \pm 5.2$ years, $P=0.077)$, baseline indexed AVA (AVAi) $(0.50 \pm 0.13$ versus $0.49 \pm 0.12$ $\left.\mathrm{cm} / \mathrm{m}^{2}, P=0.561\right)$, Logistic Euro score (8.3 [2.3-29.3] versus 7.4 [2.2-44.0], $P=0.445)$, or STS risk score $(5.2$ [1.4.-18] versus 5.0 [1.7-12.7], $P=0.887)$. There were no significant differences among the groups with regard to other baseline characteristics, indicating that the demographics of the analyzed patients were representative of the larger population.

Baseline patient characteristics and echocardiographic parameters are shown in Tables I, II. The BSA was significantly higher in men $\left(1.6 \pm 0.1 \mathrm{~m}^{2}\right)$ than in women $\left(1.4 \pm 0.2 \mathrm{~m}^{2}, P<0.001\right)$, and the serum creatinine was significantly higher in men $(1.1[0.5-2.1] \mathrm{mg} / \mathrm{dL})$ than in women $(0.8[0.5-1.6] \mathrm{mg} / \mathrm{dL}, P=0.005)$. The proportion of patients with the chronic obstructive pulmonary disease (COPD) was significantly higher in men $(14 \%)$ than in women $(2 \%, P=0.015)$. The New York Heart Association (NYHA) class was significantly lower in men $(2.0 \pm 0.5)$ than in women $(2.4 \pm 1.8, P=0.026)$.

Baseline echocardiographic measurements: At baseline, although the AVAi was significantly lower in men $(0.46 \pm$ $\left.0.02 \mathrm{~cm}^{2} / \mathrm{m}^{2}\right)$ than in women $\left(0.53 \pm 0.12 \mathrm{~cm}^{2} / \mathrm{m}^{2}, P=\right.$ $0.013)$, there were no significant differences between the groups in aortic maximum and mean pressure gradient (Table II). Women with severe AS had higher RWT (0.73 \pm 0.15 versus $0.64 \pm 0.11, P<0.001)$ than men, with smaller indexed LV end-diastolic (LVEDVi) $(50.2 \pm 13.3$ versus $\left.61.4 \pm 20.7 \mathrm{~mL} / \mathrm{m}^{2}, P=0.001\right)$ and end-systolic (LVESVi; $17.9 \pm 8.7$ versus $24.3 \pm 13.8 \mathrm{~mL} / \mathrm{m}^{2}, P=$ 0.006) volumes (Figure 2). In contrast, men with severe AS had higher LVMi $(173.2 \pm 44.6$ versus $152.3 \pm 35.4$ $\left.\mathrm{g} / \mathrm{m}^{2}, P=0.005\right)$ and lower A-wave peak velocities $(1.0 \pm$ 0.2 versus $1.2 \pm 0.3 \mathrm{~m} / \mathrm{second}, P<0.001)$ than women (Table II). There were no marked sex differences in patterns of remodeling $(P=0.070)$ : concentric hypertrophy (81\% of males and $95 \%$ of females) was seen in both sexes, but concentric remodeling tended to be seen more in men $(17 \%)$ than in women $(3 \%)$. Aortic regurgitation $(A R)>$ grade 3 was seen significantly more in men $(17 \%)$ than in women $(2 \%, P=0.005)$. There was no significant difference between men and women in terms of LVEF $(62.4 \pm 9.3$ versus $65.5 \pm 8.1, P=0.075)$, mitral regurgitation $(\mathrm{MR})(>\operatorname{grade} 3)$ ( $2 \%$ versus $8 \%, P=0.182$ ), or tricuspid max pressure gradient $(28.3 \pm 8.7$ versus $27.2 \pm$ 7.3, $P=0.537)$.

Echocardiographic assessment 3 months after TAVI: There was a marked improvement in aortic valve obstruction in both men and women (AVA $0.46 \pm 0.02$ to $1.21 \pm$ $0.30 \mathrm{~cm}^{2} / \mathrm{m}^{2}$ in men, $0.53 \pm 0.12$ to $1.25 \pm 0.34 \mathrm{~cm}^{2} / \mathrm{m}^{2}$ in women; mean pressure gradient $53.9 \pm 18.1$ to $10.6 \pm 2.0$ $\mathrm{mmHg}$ in men, $54.8 \pm 19.6$ to $12.2 \pm 5.5 \mathrm{mmHg}$ in women; peak pressure gradient $91.9 \pm 30.0$ to $21.3 \pm 7.2$ $\mathrm{mmHg}$ in men, $91.4 \pm 27.0$ to $24.8 \pm 11.2 \mathrm{mmHg}$ in women; $P<0.001$ ) (Table II) 3 months after TAVI. No patients of either sex had severe PPM. The rate of changes in echocardiographic parameters are presented in Table II. Women $(-6.0 \% \pm 14.4 \%)$ had higher reduction in the rate of change of RWT than did men $(4.4 \% \pm$ $19.0 \%, P=0.003)$. Men had higher reduction in the rate of change of LVDd $(-4.5 \% \pm 10.2 \%$ in men versus $2.6 \%$ $\pm 10.7 \%$ in women, $P=0.001)$ and LVEDVi $(-8.9 \% \pm$ $3.9 \%$ in men versus $1.5 \% \pm 3.3 \%$ in women, $P=0.045)$ than did women. LV volume reduction occurred regardless of the baseline level of the LV volume, although LV volume reduction was greatest in those patients with the highest LVEDVi and LVESVi at baseline (Figure 2). Improvement of LVEF occurred regardless of the baseline level of LVEF, although LVEF improvement was greatest in those patients with lower LVEF at baseline. Incidence of LV reverse remodeling was significantly higher in men $(50 \%)$ than in women $(26 \%, P=0.013)$. Men $(58.2 \pm$ $\left.45.2 \mathrm{~mL} / \mathrm{m}^{2}\right)$ had lower LAVi than women $(62.1 \pm 22.9$ $\left.\mathrm{mL} / \mathrm{m}^{2}, P=0.007\right) 3$ months after TAVI. There were no marked sex differences in the pattern of remodeling after TAVI $(P=0.291)$ : concentric hypertrophy (male, $76 \%$; female, $88 \%$ ) was predominantly seen in both sexes, but one male and four females changed from concentric hypertrophy to concentric remodeling. There was no sexrelated difference in paravalvular leakage (PVL) score $(9.4 \% \pm 6.4 \%$ in men versus $12.2 \% \pm 7.4 \%$ in women, $P$ $=0.096)$. Figure 3 shows representative echocardiography images before and after TAVI in each group.

\section{Discussion}

To the best of our knowledge, this study is the first to demonstrate the influence of sex on differences in car- 
Table I. Baseline Clinical Characteristics

\begin{tabular}{|c|c|c|c|c|}
\hline & $\begin{array}{c}\text { Total } \\
(n=100)\end{array}$ & $\begin{array}{c}\text { Men } \\
(n=42)\end{array}$ & $\begin{array}{l}\text { Women } \\
(n=58)\end{array}$ & $P$ value \\
\hline Age, years & $83.2 \pm 5$ & $82.4 \pm 6$ & $83.1 \pm 4$ & 0.139 \\
\hline Length of hospital stay, days & 20 [9-208] & $16[9-143]$ & $23[9-208]$ & 0.900 \\
\hline $\mathrm{BSA}, \mathrm{m}^{2}$ & $1.5 \pm 0.2$ & $1.6 \pm 0.1$ & $1.4 \pm 0.2$ & $<0.001$ \\
\hline HF hospitalization, $n(\%)$ & $37(37)$ & $14(33)$ & $23(40)$ & 0.518 \\
\hline NYHA class (median) & $2.2 \pm 0.7$ & $2.0 \pm 0.5$ & $2.4 \pm 1.8$ & 0.026 \\
\hline \multicolumn{5}{|l|}{ Comorbidities } \\
\hline Dyslipidemia, $n(\%)$ & $52(52)$ & $18(43)$ & $34(59)$ & 0.119 \\
\hline Diabetes, $n(\%)$ & $43(43)$ & $22(52)$ & $21(36)$ & 0.107 \\
\hline Hypertension, $n(\%)$ & $83(83)$ & $34(80)$ & $49(84)$ & 0.642 \\
\hline $\mathrm{CKD}, n(\%)$ & $54(54)$ & $26(62)$ & $28(48)$ & 0.177 \\
\hline Atrial fibrillation, $n(\%)$ & $27(27)$ & $12(29)$ & $15(26)$ & 0.763 \\
\hline $\mathrm{CAD}, n(\%)$ & $40(40)$ & $13(31)$ & $27(47)$ & 0.116 \\
\hline Previous myocardial infarction, $n(\%)$ & $4(4)$ & $1(2)$ & $3(5)$ & 0.482 \\
\hline CABG, $n(\%)$ & $4(4)$ & $3(7)$ & $1(2)$ & 0.172 \\
\hline $\mathrm{PAD}, n(\%)$ & $23(23)$ & $11(26)$ & $12(21)$ & 0.519 \\
\hline Carotid stenosis $>50 \%, n(\%)$ & $8(8)$ & $5(12)$ & $3(5)$ & 0.207 \\
\hline Cerebrovascular disease, $n(\%)$ & $24(24)$ & $8(19)$ & $16(28)$ & 0.324 \\
\hline COPD, $n(\%)$ & $7(7)$ & $6(14)$ & $1(2)$ & 0.015 \\
\hline \multicolumn{5}{|l|}{ Drug history } \\
\hline ACE-I/ARB, $n(\%)$ & $65(65)$ & $28(67)$ & $37(64)$ & 0.766 \\
\hline Beta-blocker, $n(\%)$ & $52(52)$ & $22(52)$ & $30(52)$ & 0.948 \\
\hline Calcium channel blocker, $n(\%)$ & $43(43)$ & $15(36)$ & $28(48)$ & 0.211 \\
\hline Diuretics, $n(\%)$ & $47(47)$ & $18(43)$ & $29(50)$ & 0.480 \\
\hline \multicolumn{5}{|l|}{ Risk scores } \\
\hline Logistic Euro score, \% & $8.3[2.3-29.3]$ & $7.0[2.3-26.1]$ & $9.8[3.6-29.3]$ & 0.106 \\
\hline STS score, $\%$ & $5.2[1.4-18]$ & $4.9[1.4-16.0]$ & $5.3[2.8-18]$ & 0.501 \\
\hline \multicolumn{5}{|l|}{ Blood } \\
\hline Hemoglobin, g/dL & $11.3 \pm 1.6$ & $11.6 \pm 1.9$ & $11.1 \pm 11.3$ & 0.187 \\
\hline Platelet, $\times 10^{4} / \mu \mathrm{L}$ & $18.6 \pm 6.0$ & $17.9 \pm 5.8$ & $19.1 \pm 6.2$ & 0.323 \\
\hline $\mathrm{HbA1C}, \%$ & $6.0 \pm 0.7$ & $6.0 \pm 0.6$ & $6.1 \pm 0.8$ & 0.456 \\
\hline Creatinine, $\mathrm{mg} / \mathrm{dL}$ & $0.9[0.5-2.1]$ & $1.1[0.5-2.1]$ & $0.8[0.5-1.6]$ & 0.005 \\
\hline $\mathrm{BNP}, \mathrm{pg} / \mathrm{mL}$ & $292[24-2834]$ & $341[24-2834]$ & $271[41-2084]$ & 0.140 \\
\hline Pulmonary hypertension, $n(\%)$ & $23(23)$ & $9(22)$ & $14(24)$ & 0.662 \\
\hline \multicolumn{5}{|l|}{ Type of approach } \\
\hline Transfemoral, $n(\%)$ & $83(83)$ & $35(82)$ & $48(83)$ & 0.817 \\
\hline Trans-apical, $n(\%)$ & $15(15)$ & $6(14)$ & $9(15)$ & 0.865 \\
\hline Trans-aortic, $n(\%)$ & $2(2)$ & $1(2)$ & $1(2)$ & 0.818 \\
\hline Type of biological valve & & & & 0.766 \\
\hline SAPIEN & $83(83)$ & $36(86)$ & $47(81)$ & 0.539 \\
\hline CoreValve/Evolut & $17(17)$ & $6(14)$ & $11(19)$ & \\
\hline
\end{tabular}

BSA indicates body surface area; HF, heart failure; NYHA, New York Heart Association classification; CKD, chronic kidney disease; $\mathrm{CAD}$, coronary artery disease; $\mathrm{CABG}$, coronary artery bypass graft; $\mathrm{PAD}$, peripheral artery disease; COPD, chronic obstructive pulmonary disease; ACE-I, angiotensin-converting enzyme inhibitor; ARB, angiotensin II receptor blocker; STS, society of thoracic surgeons predicted risk of mortality; and BNP, brain natriuretic peptide.

diac remodeling in AS and in reverse remodeling after TAVI in a Japanese population. We found that women had higher RWT with a smaller LV volume, whereas men were prone to the development of a larger LV volume and increased LVM. After TAVI, LV volume reduction was observed only in men and RWT regression was seen only in women.

Current guidelines require that, for consideration of SAVR or TAVI in patients with severe AS, symptoms related to the valvular disease be present. ${ }^{16)}$ TAVI has recently emerged as an alternative treatment for severe symptomatic AS in patients who are not suitable for surgery or are at high surgical risk. Among patients undergoing TAVI, women more commonly have insidious symptoms, resulting in late presentation and less frequent refer- ral for intervention, as well as a greater prevalence of coexisting valve diseases such as severe MR, than males. ${ }^{17)}$ Differences also exist in other comorbidities; women with AS are older and frailer, with a porcelain aorta and a greater predicted risk using both the Logistic Euro and STS risk scores. In contrast, women have a lower rate of coronary artery disease, previous myocardial revascularization, atrial fibrillation, and diabetes. ${ }^{17,18)}$

It has been reported that severe AS patients receiving TAVI in Japan are much older than those in Europe and the United States. ${ }^{19,20)}$ The population of patients in this trial had an average age approximately more than five years older than the populations in recent TAVI trials. ${ }^{6,9,11)}$ Moreover, in contrast to other studies evaluating sex in $\mathrm{AS}$, our male and female groups were similar in terms of 
Table II. Echocardiographic Data Before and After TAVI, Grouped according to Sex

\begin{tabular}{|c|c|c|c|c|}
\hline & Total $(n=100)$ & $\operatorname{Men}(n=42)$ & Women $(n=58)$ & $\begin{array}{l}P \text { value for sex } \\
\text { difference }\end{array}$ \\
\hline \multicolumn{5}{|l|}{ Aortic maximum pressure gradient $(\mathrm{mmHg})$} \\
\hline Preintervention & $91.6 \pm 28.1$ & $91.9 \pm 30.0$ & $91.4 \pm 27.0$ & 0.939 \\
\hline Postintervention & $23.2 \pm 9.9$ & $21.3 \pm 7.2$ & $24.8 \pm 11.2$ & 0.080 \\
\hline Rate of change (\%) & $-72.7 \pm 14.4$ & $-75.3 \pm 10.6$ & $-70.8 \pm 16.4$ & 0.121 \\
\hline \multicolumn{5}{|l|}{ Aortic mean pressure gradient $(\mathrm{mmHg})$} \\
\hline Preintervention & $54.4 \pm 18.9$ & $53.9 \pm 18.1$ & $54.8 \pm 19.6$ & 0.806 \\
\hline Postintervention & $11.5 \pm 4.9$ & $10.6 \pm 2.0$ & $12.2 \pm 5.5$ & 0.107 \\
\hline Rate of change $(\%)$ & $-76.7 \pm 12.9$ & $-78.6 \pm 9.9$ & $-75.3 \pm 14.7$ & 0.273 \\
\hline \multicolumn{5}{|l|}{ Aortic valve area index $\left(\mathrm{cm}^{2} / \mathrm{m}^{2}\right)$} \\
\hline Preintervention & $0.50 \pm 0.13$ & $0.46 \pm 0.02$ & $0.53 \pm 0.12$ & 0.013 \\
\hline Postintervention & $1.23 \pm 0.32$ & $1.21 \pm 0.30$ & $1.25 \pm 0.34$ & 0.506 \\
\hline Rate of change $(\%)$ & $158.3 \pm 83.4$ & $171.3 \pm 89.7$ & $148.7 \pm 77.9$ & 0.184 \\
\hline \multicolumn{5}{|l|}{ Aortic regurgitation $>3, n(\%)$} \\
\hline Preintervention & & $7(17)$ & $1(2)$ & 0.005 \\
\hline \multicolumn{5}{|l|}{ PVL score $(\%)$} \\
\hline Postintervention & & $9.4 \pm 6.4$ & $12.2 \pm 7.4$ & 0.096 \\
\hline \multicolumn{5}{|l|}{ Septum wall thickness at end-diastole (mm) } \\
\hline Preintervention & $14.6 \pm 1.8$ & $14.8 \pm 1.8$ & $14.5 \pm 1.8$ & 0.545 \\
\hline Postintervention & $14.2 \pm 2.1$ & $14.5 \pm 2.2$ & $13.9 \pm 2.1$ & 0.150 \\
\hline Rate of change $(\%)$ & $-3.1 \pm 10.2$ & $-1.5 \pm 10.2$ & $-4.2 \pm 10.1$ & 0.177 \\
\hline \multicolumn{5}{|l|}{ Posterior wall thickness at end-diastole (mm) } \\
\hline Preintervention & $13.6 \pm 1.5$ & $13.7 \pm 1.6$ & $13.4 \pm 1.4$ & 0.336 \\
\hline Postintervention & $13.0 \pm 1.6$ & $13.4 \pm 1.7$ & $12.7 \pm 1.4$ & 0.028 \\
\hline Rate of change (\%) & $-3.4 \pm 10.0$ & $-1.7 \pm 10.4$ & $-4.7 \pm 9.7$ & 0.148 \\
\hline \multicolumn{5}{|l|}{ RWT } \\
\hline Preintervention & $0.64 \pm 0.12$ & $0.64 \pm 0.11$ & $0.73 \pm 0.15$ & $<0.001$ \\
\hline Postintervention & $0.62 \pm 0.11$ & $0.66 \pm 0.13$ & $0.69 \pm 0.13$ & 0.303 \\
\hline Rate of change (\%) & $-1.6 \pm 17.2$ & $4.4 \pm 19.0$ & $-6.0 \pm 14.4$ & 0.003 \\
\hline \multicolumn{5}{|l|}{$\operatorname{LVMi}\left(\mathrm{g} / \mathrm{m}^{2}\right)$} \\
\hline Preintervention & $161.3 \pm 40.7$ & $173.2 \pm 44.6$ & $152.3 \pm 35.4$ & 0.005 \\
\hline Postintervention & $152.2 \pm 38.5$ & $157.2 \pm 38.8$ & $147.9 \pm 38.1$ & 0.230 \\
\hline Rate of change $(\%)$ & $-4.5 \pm 18.0$ & $-8.1 \pm 14.5$ & $-1.9 \pm 19.8$ & 0.089 \\
\hline \multicolumn{5}{|l|}{ Pattern of remodeling (preintervention), $n(\%)$} \\
\hline Normal geometry & 0 & 0 & 0 & 0.070 \\
\hline Concentric remodeling & $9(9)$ & $7(17)$ & $2(3)$ & \\
\hline Concentric hypertrophy & $89(89)$ & $34(81)$ & $55(95)$ & \\
\hline Eccentric hypertrophy & $2(2)$ & $1(2)$ & $1(2)$ & \\
\hline \multicolumn{5}{|c|}{ Pattern of remodeling (postintervention), $n(\%)$} \\
\hline Normal geometry & 0 & 0 & 0 & 0.291 \\
\hline Concentric remodeling & $14(14)$ & $8(19)$ & $6(10)$ & \\
\hline Concentric hypertrophy & $83(83)$ & $32(76)$ & $51(88)$ & \\
\hline Eccentric hypertrophy & $3(3)$ & $2(5)$ & $1(2)$ & \\
\hline \multicolumn{5}{|l|}{ LVDd (mm) } \\
\hline Preintervention & $43.2 \pm 6.5$ & $47.1 \pm 5.8$ & $40.4 \pm 5.6$ & $<0.001$ \\
\hline Postintervention & $42.7 \pm 5.6$ & $44.7 \pm 5.4$ & $41.2 \pm 5.3$ & 0.002 \\
\hline Rate of change (\%) & $-0.3 \pm 11.0$ & $-4.5 \pm 10.2$ & $2.6 \pm 10.7$ & 0.001 \\
\hline \multicolumn{5}{|l|}{ LVDs (mm) } \\
\hline Preintervention & $27.8 \pm 6.7$ & $30.7 \pm 6.8$ & $25.6 \pm 5.8$ & $<0.001$ \\
\hline Postintervention & $26.1 \pm 5.0$ & $27.8 \pm 4.8$ & $24.8 \pm 4.8$ & 0.003 \\
\hline Rate of change (\%) & $-3.9 \pm 15.9$ & $-7.1 \pm 17.8$ & $-1.5 \pm 14.0$ & 0.081 \\
\hline \multicolumn{5}{|l|}{ LVEDVi $\left(\mathrm{mL} / \mathrm{m}^{2}\right)$} \\
\hline Preintervention & $54.9 \pm 17.6$ & $61.4 \pm 20.7$ & $50.2 \pm 13.3$ & $<0.001$ \\
\hline Postintervention & $51.1 \pm 16.4$ & $53.3 \pm 16.5$ & $49.5 \pm 12.5$ & 0.194 \\
\hline Rate of change (\%) & $-2.9 \pm 25.8$ & $-8.9 \pm 26.3$ & $1.5 \pm 24.8$ & 0.045 \\
\hline \multicolumn{5}{|l|}{$\operatorname{LVESVi}\left(\mathrm{mL} / \mathrm{m}^{2}\right)$} \\
\hline Preintervention & $20.6 \pm 11.5$ & $24.3 \pm 13.8$ & $17.9 \pm 8.7$ & 0.006 \\
\hline Postintervention & $17.9 \pm 7.2$ & $19.1 \pm 7.4$ & $17.1 \pm 6.9$ & 0.165 \\
\hline Rate of change (\%) & $-2.0 \pm 35.8$ & $-9.5 \pm 37.8$ & $3.4 \pm 33.5$ & 0.075 \\
\hline Reduction in LVESV of $>15 \%, n(\%)$ & $36(36)$ & $21(50)$ & $15(26)$ & 0.031 \\
\hline \multicolumn{5}{|l|}{$\operatorname{LVEF}(\%)$} \\
\hline Preintervention & $64.2 \pm 8.8$ & $62.4 \pm 9.3$ & $65.5 \pm 8.1$ & 0.075 \\
\hline
\end{tabular}


Table II. Echocardiographic Data Before and After TAVI, Grouped according to Sex (Continued)

\begin{tabular}{|c|c|c|c|c|}
\hline & Total $(n=100)$ & $\operatorname{Men}(n=42)$ & Women $(n=58)$ & $\begin{array}{l}P \text { value for sex } \\
\text { difference }\end{array}$ \\
\hline Postintervention & $65.4 \pm 6.4$ & $64.5 \pm 6.5$ & $66.1 \pm 6.3$ & 0.237 \\
\hline Rate of change (\%) & $3.3 \pm 13.9$ & $5.4 \pm 17.2$ & $1.7 \pm 10.9$ & 0.346 \\
\hline \multicolumn{5}{|l|}{$\operatorname{LAVi}\left(\mathrm{mL} / \mathrm{m}^{2}\right)$} \\
\hline Preintervention & $63.5 \pm 35.6$ & $61.9 \pm 43.9$ & $64.8 \pm 28.4$ & 0.223 \\
\hline Postintervention & $60.7 \pm 33.3$ & $58.2 \pm 45.2$ & $62.1 \pm 22.9$ & 0.007 \\
\hline Rate of change (\%) & $-1.1 \pm 25.2$ & $-4.1 \pm 27.6$ & $1.1 \pm 23.2$ & 0.312 \\
\hline \multicolumn{5}{|l|}{$\mathrm{E}(\mathrm{m} / \mathrm{second})$} \\
\hline Preintervention & $0.9 \pm 0.3$ & $0.9 \pm 0.3$ & $0.9 \pm 0.3$ & 0.908 \\
\hline Postintervention & $0.9 \pm 0.3$ & $0.9 \pm 0.3$ & $0.9 \pm 0.3$ & 0.504 \\
\hline Rate of change (\%) & $10.8 \pm 37.6$ & $8.1 \pm 36.5$ & $12.8 \pm 38.5$ & 0.544 \\
\hline \multicolumn{5}{|l|}{ A (m/second) } \\
\hline Preintervention & $1.1 \pm 0.3$ & $1.0 \pm 0.2$ & $1.2 \pm 0.3$ & $<0.001$ \\
\hline Postintervention & $1.2 \pm 0.3$ & $1.1 \pm 0.2$ & $1.3 \pm 0.3$ & 0.002 \\
\hline Rate of change (\%) & $8.1 \pm 34.6$ & $9.7 \pm 45.3$ & $7.1 \pm 26.3$ & 0.751 \\
\hline \multicolumn{5}{|l|}{$\mathrm{E} / \mathrm{A}$} \\
\hline Preintervention & $0.8 \pm 0.5$ & $0.9 \pm 0.6$ & $0.7 \pm 0.4$ & 0.246 \\
\hline Postintervention & $0.8 \pm 0.3$ & $0.8 \pm 0.3$ & $0.8 \pm 0.3$ & 0.718 \\
\hline Rate of change (\%) & $6.3 \pm 46.7$ & $-4.7 \pm 45.2$ & $13.3 \pm 46.8$ & 0.098 \\
\hline \multicolumn{5}{|l|}{ Deceleration time (ms) } \\
\hline Preintervention & $303.2 \pm 113.2$ & $292.6 \pm 116.4$ & $311.4 \pm 111.4$ & 0.367 \\
\hline Postintervention & $316.4 \pm 100.1$ & $315.3 \pm 113.5$ & $316.4 \pm 90.85$ & 0.592 \\
\hline Rate of change (\%) & $8.5 \pm 39.6$ & $10.4 \pm 39.9$ & $7.1 \pm 39.6$ & 0.682 \\
\hline \multicolumn{5}{|c|}{ Mitral regurgitation $>3, n(\%)$} \\
\hline Preintervention & $5(5)$ & $1(2)$ & $4(8)$ & 0.182 \\
\hline Postintervention & $4(4)$ & $1(2)$ & $3(5)$ & 0.637 \\
\hline \multicolumn{5}{|c|}{ Tricuspid max pressure gradient $(\mathrm{mmHg})$} \\
\hline Preintervention & $27.7 \pm 7.9$ & $28.3 \pm 8.7$ & $27.2 \pm 7.3$ & 0.537 \\
\hline Postintervention & $26.1 \pm 7.7$ & $26.3 \pm 7.7$ & $26.0 \pm 7.8$ & 0.844 \\
\hline Rate of change (\%) & $-4.3 \pm 33.1$ & $-9.8 \pm 38.8$ & $-0.2 \pm 27.8$ & 0.172 \\
\hline
\end{tabular}

PVL indicates paravalvular leakage; RWT, relative wall thickness; LVMi, indexed left ventricular (LV) mass; LVDd, LV end-diastolic diameter; LVDs, LV end-systolic diameter; LVEDVi, indexed LV end-diastolic volume; LVESVi, indexed LV end-systolic volume; LVEF, LV ejection fraction; and LAVi, indexed left atrial volume.

age, comorbidity, cardiac risk score, and aortic valve gradients. Only AVAi, NYHA classification, baseline AR, creatinine, and, expectedly, COPD prevalence and BSA differed between the two groups. The advanced symptomatic status of women with AS has been attributed to more severe LV diastolic dysfunction, a higher prevalence of pulmonary hypertension, severity of AS, and older age. ${ }^{21,22)}$ Although age, severity of AS, LV diastolic dysfunction, and tricuspid max pressure gradient were similar in this study, higher RWT and smaller LV volume in women might be associated with advanced symptomatic status. It is well known that smoking history is significantly higher in men with AS. ${ }^{22)}$ Although smoking status was not recorded in this study, a high history of smoking in men might be related to having a history of COPD. Higher LV cavity size and mass in men may contribute to higher prevalence of baseline AR, but the PVL after TAVI was similar between sexes in this study.

It is still debated whether sex-specific factors influence and modify the clinical course of AS over time and whether hormonal changes, including history of pregnancy and age of menopause, can impact TAVI outcomes. ${ }^{23-25)}$ Myocardial response to pressure overload is different in women and men, ${ }^{6,726)}$ and it has been reported that female patients have a higher prevalence of normal geometry and concentric remodeling, while male patients show a higher rate of maladaptive remodeling (including concentric hypertrophy and eccentric hypertrophy) that eventually leads to LV dilation. ${ }^{6,8)}$ Women also typically have higher LVEF; however, little is known about the role of gender in LV remodeling in extreme age in the Japanese population.

In this study, aortic valve gradients, RWT, and LVMi were higher at baseline than those of the past studies. ${ }^{6,11}$ Moreover, $89 \%$ of patients (male, $81 \%$; female, $95 \%$ ) displayed concentric hypertrophy before TAVI. One possible reason is that the patients' hearts in this Japanese cohort were small compared with the reference values in the ASE guidelines. ${ }^{12,27)}$ Moreover, concentric hypertrophy of the LV is considered to progress as AS patients grow older. We have demonstrated that men and women with severe AS and similar comorbidities remodel in different ways: women exhibited higher RWT with a smaller LV volume, while men were prone to the development of a larger LV volume and increased LVM. In the present study, we defined $L V$ reverse remodeling as a reduction in LVESV of $>15 \%$. The definition of LV reverse remodeling varies in the literature, but reduction in LVESV of > $15 \%$ after cardiac resynchronization therapy has been reported to be a valid measure of volume responders. ${ }^{15)}$ Incidence of LV reverse remodeling was significantly higher in men than in women after TAVI and was greatest in the patients with the highest LV volume at baseline. Similarly, 
A

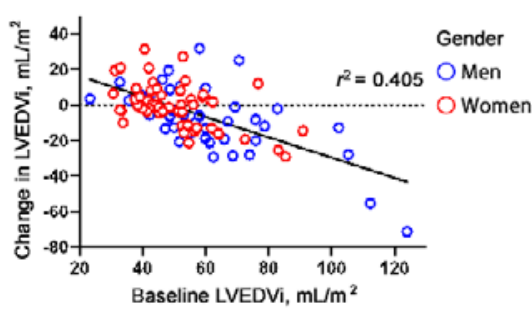

D

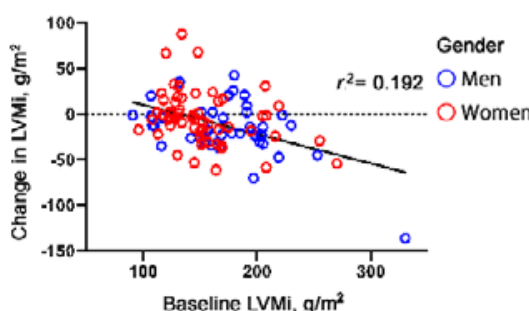

B

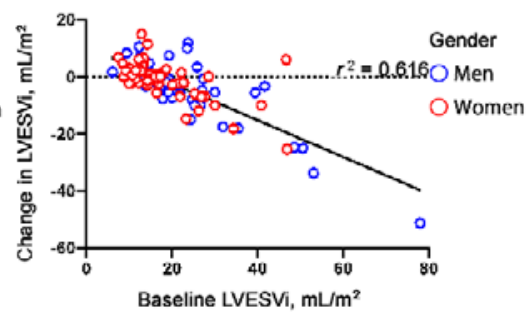

E

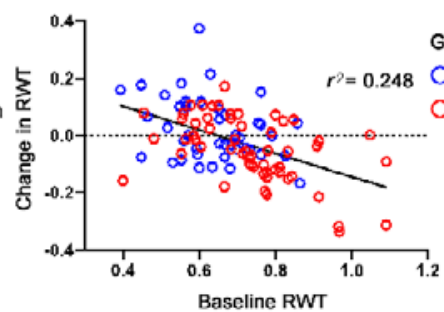

C

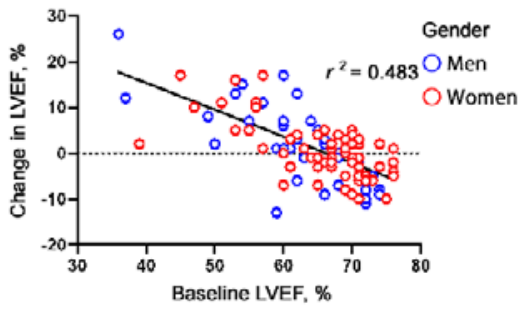

F

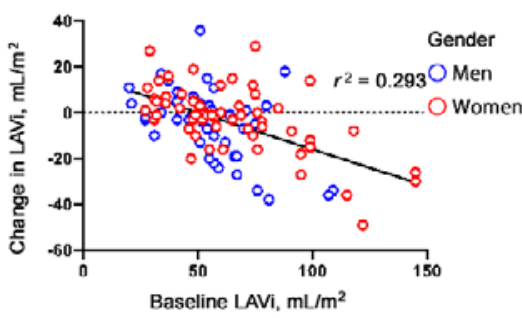

Figure 2. Relationship between cardiac reverse remodeling parameters after transcatheter aortic valve implantation and baseline parameters according to sex. A: Relationship between change in indexed left ventricular (LV) end-diastolic volume (LVEDVi) and baseline LVEDVi. B: Relationship between change in indexed LV end-systolic volume (LVESVi) and baseline LVESVi. C: Relationship between change in LV ejection fraction (LVEF) and baseline LVEF. D: Relationship between change in indexed LV mass (LVMi) and baseline LVMi. E: Relationship between change in relative wall thickness (RWT) and baseline RWT. F: Relationship between change in indexed left atrial volume (LAVi) and baseline LAVi.

Men

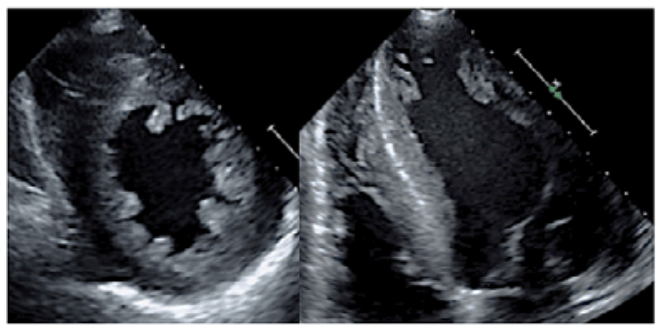

RWT 0.67

LVMi $330 \mathrm{~g} / \mathrm{m}^{2}$

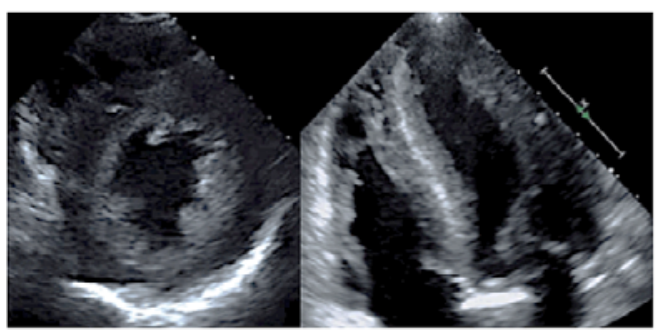

RWT 0.65

LVMi $194 \mathrm{~g} / \mathrm{m}^{2}$
LVEDVi $57 \mathrm{~mL} / \mathrm{m}^{2}$

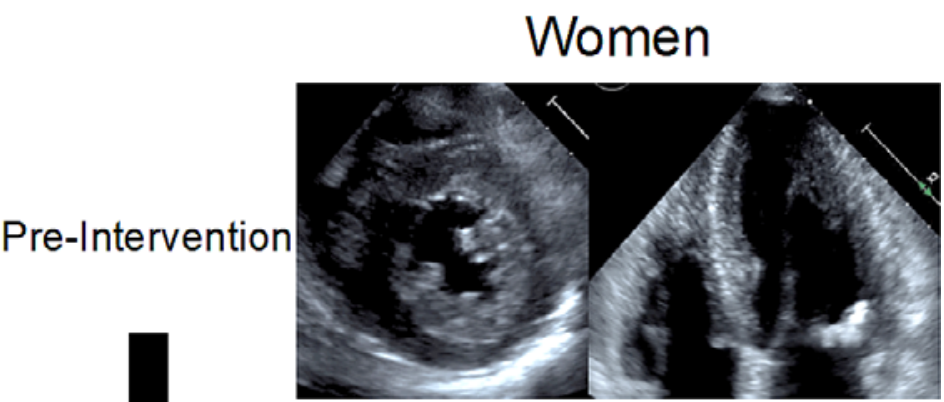

RWT 0.72

LVMi $128 \mathrm{~g} / \mathrm{m}^{2}$

LVEDVi $33 \mathrm{~mL} / \mathrm{m}^{2}$

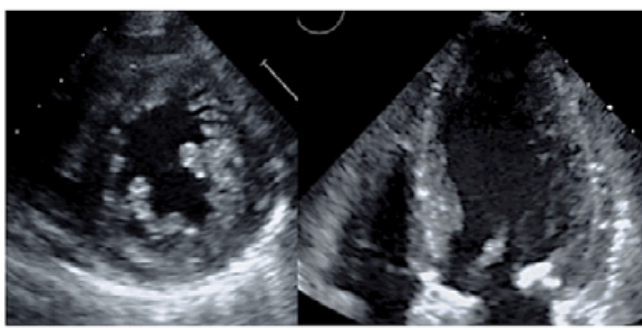

RWT 0.65

LVMi $141 \mathrm{~g} / \mathrm{m}^{2}$

LVEDVi $42 \mathrm{~mL} / \mathrm{m}^{2}$

Figure 3. Short-axis and four-chamber echocardiographic images of the left ventricle before and after transcatheter aortic valve implantation (TAVI). The left-hand panel shows a typical male pattern of remodeling with increased left ventricular (LV) cavity size and greater LV mass at baseline (top image) and then reverse remodeling 3 months after TAVI (bottom image). The right-sided panel depicts a typical female ventricle in severe aortic stenosis (AS) with a lower LV mass and a small LV cavity size (top image) and subsequent large relative wall thickness (RWT) regression 3 months after TAVI (bottom image). LVEDVi, indexed LV end-diastolic volume; LVMi, indexed LV mass 
the size of LA in men was significantly lower than that in women after TAVI. This may reflect a greater improvement in LV cavity pressure, transmitral gradient, and mitral valve tethering forces in men. On the other hand, RWT regression was seen only in women after TAVI. Thus, this study revealed that myocardial responses after TAVI differ between the sexes, as well as baseline characteristics.

Studies are currently underway to assess the benefits of expanding the use of TAVI to asymptomatic severe AS and moderate AS with reduced LVEF and are expected to have a positive outcome. ${ }^{28,29)}$ Cardiac and reverse remodeling will be different in the context of TAVI performed in these patient groups, which have a younger age. Our study results might be useful as a fundamental data in comparing reverse remodeling after the procedure for each indication of TAVI that can provide the most therapeutic effects in Japanese population.

Several limitations of our study must be considered. First, half $(52 \%)$ of the original study population did not complete the study protocol, mainly due to geographical reasons. This may have introduced bias, although the analyzed population did not differ in terms of baseline characteristics from the original population. Further studies are needed to evaluate the LV reverse remodeling using the data of high follow-up rates. Second, echocardiography was performed for the assessment of LV remodeling instead of cardiac magnetic resonance (CMR), which is the golden standard for evaluating LV remodeling. Using an echocardiography-based approach to LV remodeling can lead to a potential underestimation of biological sex dimorphism in LV remodeling in AS. $\left.{ }^{6}\right)$ However, CMR requires the suspension of respiration and gadolinium-based contrast agent. There are several factors that may limit the ability of the elderly patients to perform CMR, such as consistent breath-holds and renal dysfunction. Further studies are needed for evaluating LV remodeling using CMR. Third, the postprocedure scan was performed 3 months after TAVI. Although it is well documented that most of reverse remodeling occurs within the first 6 months, ${ }^{30)}$ this timepoint could still be too early to detect any subtle differences between the sexes. Stangl, et al. reported that LV reverse remodeling was observed both in men and women 3 months after TAVI, but improvement of LVEF was significant only in women. ${ }^{11)}$ Chen, et al. reported that women exhibited an early regression of LVMi. $^{31)}$ In contrast, the regression of LVMi in men seemed to be more gradual and the significant regression of LVMi from baseline began to be observed since 3 months later after TAVI. Further studies are needed to evaluate the LV reverse remodeling using long-term follow-up data. Finally, because of the relatively small sample size, the study may be not sufficiently powered to assess all gender differences.

\section{Conclusion}

This study demonstrates that there are clear differences in the way that male and female hearts adapt to the pressure overload of AS despite similar comorbidities and valvular gradients. Sex-related differences of reverse re- modeling after TAVI also differed, reflecting the pattern of remodeling in AS in extreme age in the Japanese population.

\section{Acknowledgments}

We thank Aya Sasaki for her technical assistance.

\section{Disclosure}

Conflicts of interest: There was no specific financial support or funding for this study.

The authors have no conflicts of interest to declare.

\section{References}

1. Nkomo VT, Gardin JM, Skelton TN, Gottdiener JS, Scott CG, Enriquez-Sarano M. Burden of valvular heart diseases: a population-based study. Lancet 2006; 368: 1005-11.

2. Rohde LE, Zhi G, Aranki SF, Beckel NE, Lee RT, Reimold SC Gender-associated differences in left ventricular geometry in patients with aortic valve disease and effect of distinct overload subsets. Am J Cardiol 1997; 80: 475-80.

3. Villari B, Campbell SE, Schneider J, Vassalli G, Chiariello M, Hess OM. Sex-dependent differences in left ventricular function and structure in chronic pressure overload. Eur Heart J 1995; 16: $1410-9$.

4. Douglas PS, Otto CM, Mickel MC, Labovitz A, Reid CL, Davis $\mathrm{KB}$. Gender differences in left ventricle geometry and function in patients undergoing balloon dilatation of the aortic valve for isolated aortic stenosis. NHLBI Balloon Valvuloplasty Registry. Br Heart J 1995; 73: 548-54.

5. Ganau A, Devereux RB, Roman MJ, et al. Patterns of left ventricular hypertrophy and geometric remodeling in essential hypertension. J Am Coll Cardiol 1992; 19: 1550-8.

6. Treibel TA, Kozor R, Fontana M, et al. Sex dimorphism in the myocardial response to aortic stenosis. JACC Cardiovasc Imaging 2018; 11: 962-73.

7. Devereux RB, Alonso DR, Lutas EM, et al. Echocardiographic assessment of left ventricular hypertrophy: comparison to necropsy findings. Am J Cardiol 1986; 57: 450-8.

8. Carroll JD, Carroll EP, Feldman T, et al. Sex-associated differences in left ventricular function in aortic stenosis of the elderly. Circulation 1992; 86: 1099-107.

9. Dobson LE, Fairbairn TA, Musa TA, et al. Sex-related differences in left ventricular remodeling in severe aortic stenosis and reverse remodeling after aortic valve replacement: a cardiovascular magnetic resonance study. Am Heart J 2016; 175: 101-11.

10. Dweck MR, Joshi S, Murigu T, et al. Left ventricular remodeling and hypertrophy in patients with aortic stenosis: insights from cardiovascular magnetic resonance. J Cardiovasc Magn Reson 2012; 14: 50

11. Stangl V, Baldenhofer G, Knebel F, et al. Impact of gender on three-month outcome and left ventricular remodeling after transfemoral transcatheter aortic valve implantation. Am J Cardiol 2012; 110: 884-90.

12. Lang RM, Bierig M, Devereux RB, et al. Recommendations for chamber quantification: a report from the American Society of Echocardiography's Guidelines and Standards Committee and the Chamber Quantification Writing Group, developed in conjunction with the European Association of Echocardiography, a branch of the European Society of Cardiology. J Am Soc Echocardiogr 2005; 18: 1440-63.

13. Mancia G, De Backer G, Dominiczak A, et al. 2007 Guidelines for the management of arterial hypertension: the Task Force for the Management of Arterial Hypertension of the European Soci- 
ety of Hypertension (ESH) and of the European Society of Cardiology (ESC). Eur Heart J 2007; 28: 1462-536.

14. Kappetein AP, Head SJ, Généreux P, et al. Updated standardized endpoint definitions for transcatheter aortic valve implantation: the Valve Academic Research Consortium-2 consensus document. Eur Heart J 2012; 33: 2403-18.

15. Stellbrink C, Breithardt OA, Franke A, et al. Impact of cardiac resynchronization therapy using hemodynamically optimized pacing on left ventricular remodeling in patients with congestive heart failure and ventricular conduction disturbances. J Am Coll Cardiol 2001; 38: 1957-65.

16. Baumgartner H, Falk V, Bax JJ, et al. 2017 ESC/EACTS Guidelines for the management of valvular heart disease. Eur Heart $\mathrm{J}$ 2017; 38: 2739-91.

17. Chandrasekhar J, Dangas G, Mehran R. Valvular heart disease in women, differential remodeling, and response to new therapies. Curr Treat Options Cardiovasc Med 2017; 19: 74.

18. Naoum C, Blanke P, Dvir D, et al. Clinical outcomes and imaging findings in women undergoing TAVR. JACC Cardiovasc Imaging 2016; 9: 483-93.

19. Yamamoto M, Watanabe $\mathrm{Y}$, Tada $\mathrm{N}$, et al; OCEAN-TAVI investigators. Transcatheter aortic valve replacement outcomes in Japan. Optimized CathEter vAlvular iNtervention (OCEAN) Japanese multicenter registry. Cardiovasc Revasc Med 2019; 20: 843-51.

20. Kamioka N, Arita T, Hanyu M, et al. Valve hemodynamics and clinical outcomes after transcatheter aortic valve replacement for a small aortic annulus. Int Heart J 2019; 60: 86-92.

21. Fuchs C, Mascherbauer J, Rosenhek R, et al. Gender differences in clinical presentation and surgical outcome of aortic stenosis. Heart 2010; 96: 539-45.

22. Toyofuku M, Taniguchi T, Morimoto T, et al. Sex differences in severe aortic stenosis- clinical presentation and mortality. Circ J 2017; 81: 1213-21.

23. Scheuer J, Malhotra A, Schaible TF, Capasso J. Effects of gonadectomy and hormonal replacement on rat hearts. Circ Res 1987; 61: 12-9.

24. Chieffo A, Petronio AS, Mehilli J, et al. 1-year clinical outcomes in women after transcatheter aortic valve replacement: results from the first WIN-TAVI Registry. JACC Cardiovasc Interv 2018; 11: 1-12.

25. Piro M, Della Bona R, Abbate A, Biasucci LM, Crea F. Sexrelated differences in myocardial remodeling. J Am Coll Cardiol 2010; 55: 1057-65.

26. Nara Y, Watanabe Y, Kataoka A, et al. Incidence, predictors, and midterm clinical outcomes of myocardial injury after transcatheter aortic-valve implantation. Int Heart J 2018; 59: 1296-302.

27. Daimon M, Watanabe H, Abe Y, et al. Normal values of echocardiographic parameters in relation to age in a healthy Japanese population: the JAMP study. Circ J 2008; 72: 1859-66.

28. Spitzer E, Van Mieghem NM, Pibarot P, et al. Rationale and design of the transcatheter aortic valve replacement to UNload the left ventricle in patients with ADvanced heart failure (TAVR UNLOAD) trial. Am Heart J 2016; 182: 80-8.

29. Lancellotti P, Magne J, Dulgheru R, et al. Outcomes of patients with asymptomatic aortic stenosis followed up in heart valve clinics. JAMA Cardiol 2018; 3: 1060-8.

30. Gelsomino S, Frassani R, Morocutti G, et al. Time course of left ventricular remodeling after stentless aortic valve replacement. Am Heart J 2001; 142: 556-62.

31. Chen SC, Leu HB, Chang HH, et al. Women had favourable reverse left ventricle remodelling after TAVR. Eur J Clin Invest 2020; 50: e13183. 